\title{
Histological studies in primary neuritic leprosy: changes in the apparently normal skin
}

\author{
SUJAI SUNEETHA*, S. ARUNTHATHI*, \\ SUSHIL CHANDI\#, NISHA KURIAN* \& \\ CHINOY J. G. CHACKO* \\ *Schieffelin Leprosy Research and Training Centre, Karigiri, Tamil \\ Nadu-632 106, India; \#Christian Medical College and Hospital, \\ Vellore, Tamil Nadu-632 004, India
}

Accepted for publication 10 July 1998

Summary The visually normal skin of 196 patients diagnosed clinically to have primary neuritic leprosy was studied histologically to determine whether there were any specific changes due to the disease in this site. Histological changes due to leprosy were seen in $32.1 \%$ of the patients, and included, indeterminate leprosy in $19.4 \%$, borderline tuberculoid leprosy in $6.6 \%$ and borderline lepromatous leprosy in $6 \cdot 1 \%$. The remaining biopsies showed mild non-specific dermal inflammation, mild nerve changes or no significant lesion. The nerve inflammation and/or granulomas were mostly in the deep dermal nerves or neurovascular complexes. This study shows that there is a cutaneous component to primary neuritic leprosy and the disease is not totally confined to nerves. The absence of visible hypopigmented patches in these patients is probably related to the deep location of the dermal inflammation.

\section{Introduction}

Primary neuritic leprosy (PNL) presents as a peripheral neuropathy with either pure sensory or combined sensory and motor nerve dysfunction. The characteristic clinical skin lesions of leprosy are absent. ${ }^{1}$ No definite information is available as to the extent of involvement of the apparently normal skin in PNL. Histological studies of skin in PNL are $\mathrm{few}^{2,3}$ and have only suggested mild dermal nerve involvement in the skin. An earlier study of skin biopsies from PNL patients at Karigiri has shown changes consistent with leprosy in 11 out of 17 patients, including two with a borderline tuberculoid (BT) and one with a borderline lepromatous (BL) histology. ${ }^{4}$ This prompted us to make a more extensive study of the histological changes in the skin of PNL patients even though they do not have clinically visible skin lesions. India

Correspondence to: S. Suneetha, Director, Dhoolpet Leprosy Research Centre, Karwan Post, Hyderabad-500 006, 


\section{Materials and methods}

A total of 196 patients seen at the Schieffelin Leprosy Research and Training Centre, Karigiri between 1978 and 1991 were clinically classified as PNL based on the following criteria: sensory or sensory and motor dysfunction, negative skin smear and no detectable skin lesions. ${ }^{5}$ A diagnostic cutaneous nerve biopsy was performed on all these patients (radial cutaneous nerve, 94; musculocutaneous nerve, 73; ulnar cutaneous nerve, 15 and sural nerve, 14). Histological confirmation of leprosy was present in 158 patients. Although the remaining 38 patients had no nerve biopsy confirmation, they were classified and treated as PNI, since they had definite nerve thickening with sensory or sensory and motor nerve deficit. ${ }^{6}$ Other causes for a peripheral neuropathy, such as congenital, traumatic and diabetic neuropathy, were excluded in these patients.

Biopsies were usually taken from the skin over the cutaneous nerve selected for biopsy to establish a diagnosis of PNL. An elliptical piece of skin was taken from the edge of the skin incision. This was sometimes within the area of sensory change and on occasions outside it. Skin biopsies were also obtained from other parts of the body that had sensory changes such as anaesthesia, hypoaesthesia, paraesthesia or dryness. The biopsied skin samples were fixed in formal-Zenker's fixative for $4-6 \mathrm{~h}$ and then transferred to $70 \%$ alcohol. They were subsequently routinely processed and embedded in paraf fin. Serial $5 \mu \mathrm{m}$ sections were cut and stained with haematoxylin and eosin and a modified Fite's stain for acid fast bacilli. ${ }^{7}$ In all, 182 of these patients were followed up for varying periods ranging from 6 months to 12 years to look for any changes in the pattern of the disease.

\section{Results}

A total of 196 patients clinically diagnosed as PNL were studied. Skin biopsies were taken from the nerve biopsy site in 147 patients $(75 \cdot 3 \%)$ and from other sites in 49 patients $(24 \cdot 7 \%)$. These were from areas of anaesthesia in 133 patients and from areas of normal sensation in 63 patient s. Their histological classification is given in Table 1 .

\section{INDETERMINATE LEPROSY}

The skin biopsies of 38 patients $(19.4 \%)$ in the study showed changes of indeterminate leprosy. The deep dermal nerves were usually enlarged and showed definite intraneural

Table 1. Histological classification of skin

\begin{tabular}{lc}
\hline Classification & No. of patients $(\%)$ \\
\hline Indeterminate & $38(19 \cdot 4)$ \\
Consistent with indeterminate leprosy & $52(26 \cdot 5)$ \\
Borderline tuberculoid leprosy & $13(6 \cdot 6)$ \\
Borderline lepromatous leprosy & $12(6 \cdot 1)$ \\
Non-specific inflammation & $44(22 \cdot 5)$ \\
No significant lesion & $37(18 \cdot 9)$ \\
Total & 196 \\
\hline
\end{tabular}




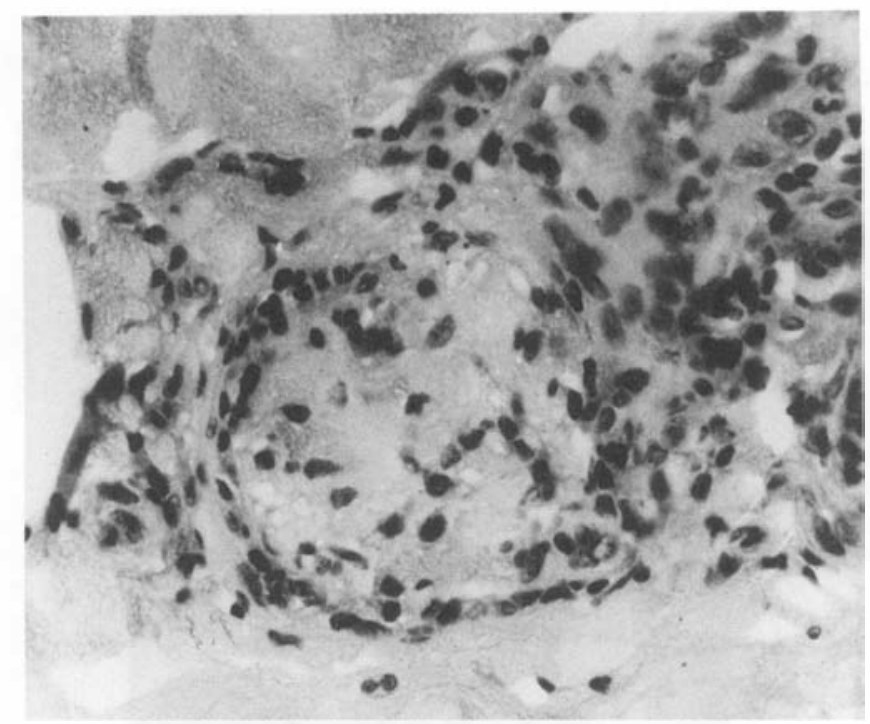

Figure 1. Indeterminate leprosy, showing a deep dermal nerve with a few intraneural lymphocytes and dense perineurial inflammation $(H \& E \times 200)$.

infiltration by lymphocytes (Figure 1). Additionally, 66\% showed perineural inflammation with lymphocytes and histiocytes. Associated mild perivascular and adnexal inflammation was common.

Acid fast bacilli were present in six cases, in nerve alone (two cases), nerve and macrophages (two), nerve and smooth muscle (one) or endothelial cells of blood vessels (one).

\section{CONSISTENT WITH INDETERMINATE LEPROSY}

This classification was ascribed to 52 skin biopsies (26.5\%) which showed mild to moderate degrees of perivascular and adnexal inflammation including smooth muscle involvement and enlarged deep dermal nerves with perineurial lymphohistiocytic infiltration. Acid fast bacilli were not seen.

\section{BORDERLINE TUBERCULOID LEPROSY}

The biopsied skin of 13 patients (6.6\%) showed histological features of borderline tuberculoid leprosy. These consisted of epithelioid cell granulomas involving neurovascular complexes in the deep dermis and sometimes blood vessels and adnexal structures (Figure 2). The granulomas contained moderate numbers of lymphocytes either as focal collections or diffusely scattered in the granuloma. Giant cells were present in the epithelioid cell aggregates in eight of the biopsies. Nerve inflammation consisted of intraneural lymphocytes and occasionally intraneural histiocytes and epithelioid cells. An occasional acid fast bacillus was identified in the dermal nerves in five biopsies and in the endothelial cells of a blood vessel in one. 


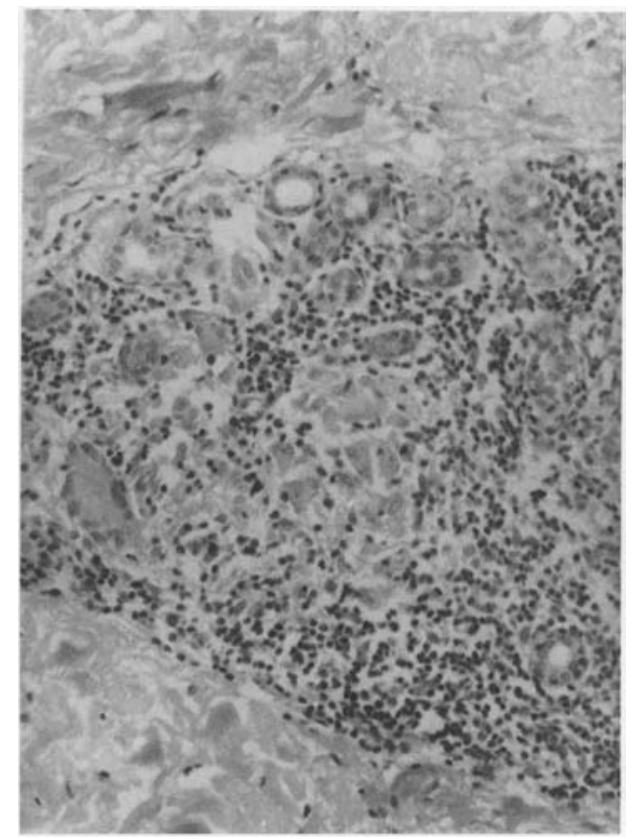

Figure 2. Borderline tuberculoid leprosy, with a dermal granuloma consisting of epithelioid cells, Langhan's giant cells and a cuff of lymphocytes $(\mathrm{H} \& \mathrm{E} \times 100)$.

BORDERLINE LEPROMATOUS LEPROSY

In 12 patients $(6 \cdot 1 \%)$, the skin revealed deep dermal nerve inflammation accompanied by collections of lymphocytes, histiocytes and macrophages close to nerves, neurovascular complexes and other skin adnexal structures (Figure 3). A few bacilli were present in nine biopsies and moderate numbers of bacilli in three biopsies. The bacilli were present in nerves and macrophages (five cases), nerves alone (four), nerves, macrophages and smooth muscle (one), in macrophages alone (one) and in endothelial cells (one).

\section{NON-SPECIFIC INFLAMMATION}

In 44 patients $(22.5 \%)$, the skin biopsies showed non-specific inflammatory changes, such as a few small perivascular lymphohistiocytic collections around upper dermal blood vessels and skin adnexal structúres. Dermal nerves were within normal limits in 37 patients (18.9\%) and were slightly enlarged with prominent perineurial cells and Schwann cell prolipheration in seven patients $(3 \cdot 6 \%)$. Acid fast bacilli were not present.

\section{NO SIGNIFICANT LESION}

In 37 patients (18.9\%) there was no evidence of dermal inflammation, neuritis or acid fast bacilli. These skin biopsies were designated as having 'no significant lesion'.

The relationship between the sensory status of the biopsied area and histological diagnosis is given in Table 2. Biopsies taken from an area of sensory change revealed 


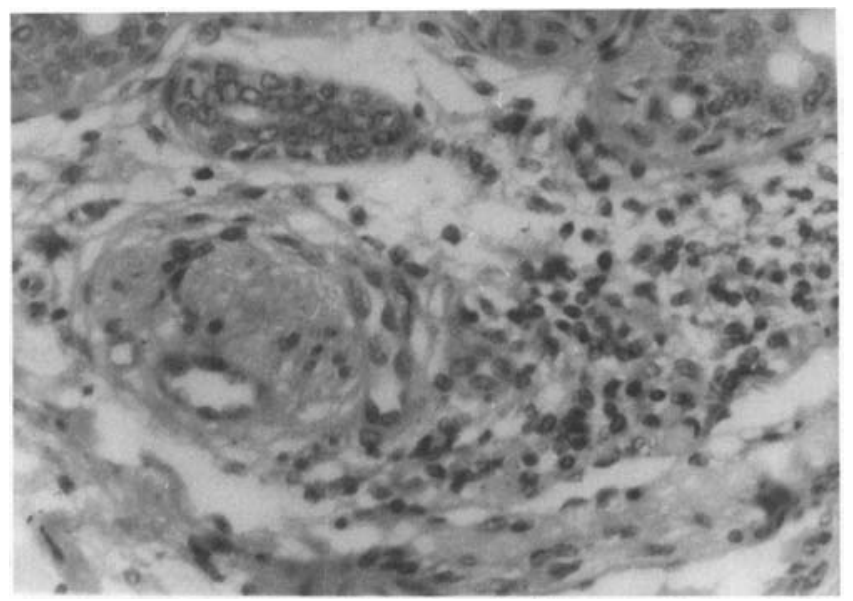

Figure 3. Borderline lepromatous leprosy, showing a macrophage granuloma involving a sweat gland complex as well as perineurial lamination and inflammation. Fite stained sections revealed acid fast bacilli in the macrophages $(\mathrm{H} \& \mathrm{E} \times 200)$.

inflammation of dermal nerves in $65.4 \%$ of the patients whereas those from an area of normal sensation showed nerve inflammation in only $44.4 \%$ of the patients $(p<0 \cdot 001)$.

Among the 182 patients followed up in the study, $29(15.9 \%)$ developed visible skin lesions during the follow-up period.

\section{Discussion}

The apparently normal skin in lepromatous, borderline, tuberculoid and indeterminate leprosy has been studied by different workers ${ }^{8-10}$ but relatively few histological studies of the skin in PNL. A few case reports and studies on small numbers have found specific changes due to leprosy in the skin in $\mathrm{PNL}^{2-4}$ but there are also reports to the contrary. ${ }^{6,11}$

In the present study, there were histological changes due to leprosy in the skin of $32.1 \%$ of the patients with PNL, though they did not have skin patches of leprosy. In all, $12.7 \%$ of the patients revealed either epithelioid or macrophage granulomas. The granulomas were located

Table 2. Relationship between anaesthesia and histology

\begin{tabular}{lccr}
\hline & \multicolumn{3}{c}{ Sensation } \\
\cline { 2 - 4 } Histological classification & Anaesthesia & Normal & Total \\
\hline Borderline lepromatous leprosy & 9 & 3 & 12 \\
Borderline tuberculoid leprosy & 11 & 2 & 13 \\
Indeterminate leprosy & 67 & 23 & 90 \\
Non-specific inflammation & 25 & 19 & 44 \\
No significant lesion & 21 & 16 & 37 \\
Total & 133 & 63 & 196 \\
& & & \\
\hline
\end{tabular}


mostly in relation to the deep and mid-dermal nerves and neurovascular complexes. This would account for the somatosensory and autonomic neuropathic manifestations of the disease $^{12,13}$ in the absence of the characteristic skin lesions. The absence of visible hypopigmented skin lesions in these patients is probably related to the deep location of the granulomas in the dermis, beyond the scope of exercising any direct influence by proximity on the melanocytes in the epidermal stratum bacillus.

It would appear that it is only when the dermal inflammation reaches the superficial dermal regions, is it likely to produce a visible skin patch. Follow-up studies suggests that this may be true, since 29 of the patients in this cohort subsequently developed skin patches. The perineurial inflammation seen in 52 patients designated 'consistent with indeterminate leprosy' and the intraneural inflammation seen in 'indeterminate leprosy' may represent steps in the progression to granuloma formation and determined forms of the disease.

The present study suggests that leprosy primarily affects the nerve from where it breaks out into the dermis to produce cutaneous lesions, with individual patients exhibiting histological features of the disease in varied forms and stages of evolution. Haematogenous spread of infection from a primary lesion in the nasal mucosa is a distinct possibility, in view of the inflammatory infiltrate predominantly involving neurovascular complexes and blood vessels. In recent years, there is increasing experimental evidence in favour of the nasal mucosa as the primary site of entry of Mycobacterium leprae. ${ }^{14,15}$

The present study has shown that a skin biopsy from an area of anaesthesia is more likely to show evidence of dermal nerve inflammation and/or granuloma than a biopsy from an area with normal sensation.

In conclusion, there are specific histological changes due to leprosy in the skin of PNL patients although they do not manifest skin patches. The deep dermal location of the inflammation may account for the absence of hypopigmented skin lesions. The presence of an inflammatory reaction in dermal nerves, with granulomas and acid fast bacilli in the skin indicates widespread dissemination of the disease, even when it clinically appears to be confined to a few major nerves.

\section{Acknowledgements}

We wish to thank Dr C. K. Job, Dr P. S. S. S. Rao and Dr Steve Withington for their advice and suggestions during the preparation of this manuscript, and Mrs Reeny and Mr K. Ramana Babu for secretarial assistance.

\section{References}

${ }^{1}$ Jacob M, Mathai R. Diagnostic efficacy of cutaneous nerve biopsy in primary neuritic leprosy. Int J Lepr, 1988; 56: $56-60$.

${ }^{2}$ Kundu KK, Ghosh S. Studies on the development of dermal changes in Primary Polyneuritic leprosy. Bull Calcutta Sch Trop Med, 1971; 19: 90-91.

${ }^{3}$ Rodriquez G, Sanchez W, Chalela JG, Soto J. Primary neuritic leprosy. J Am Acad Dermatol, 1993; 29: 10501052.

4 Pannikar VK, Arunthathi S, Chacko CJG, Fritchi EP. A clinico pathological study of primary neuritic leprosy. Ind J Lepr, 1983, 56: 56-60.

5 Noordeen SK. Epidemiology of (poly) neuritic leprosy. Lepr India, 1972; 44: 90-96.

${ }^{6}$ Kaur G, Girdhar BK, Girdhar J, Sengupta U, Desikan KV. Clinical, immunological and histological study of neuritic leprosy patients. Int J Lepr, 1991; 59: 385-391. 
${ }^{7}$ Job CK, Chacko CJG. A modification of Fite's stain for demonstration of M. leprae in tissue sections. Ind J Lepr, 1986; 58: 17-18.

${ }^{8}$ Ganapathi R, Desikan KV, Iyer CGS. Study of apparently normal skin in leprosy. Int J Lepr, 1972; 40: 281-290.

${ }^{9}$ Katoch VM, Mukher jee A, Girdhar BK. A bacteriological and histopathological study of apparently normal skin in lepromatous leprosy. Lepr India, 1980; 52: 508-512.

${ }^{10}$ Sakuntala R, Pratao VK, Sharma NK, Dayal SS, Aggarwal SK. Histological profile in apparently normal skin of leprosy patients. Lepr India, 1982; 54: 508-512.

11 Asok Kumar SKA, Reddy BSN, Ratnakar C. Correlation of skin and nerve histopathology in leprosy. Lepr Rev, 1996; 67: 119-125.

12 Gadoth N, Bechar M, Kushmir M, Davidovitz S, Sandbank U. Somatosensory and autonomic neuropathy as the only manifestation of long standing leprosy. J Neuro Sci, 1979; 43: 471-477.

13 Talwar S, Jha PK, Tiwari VD. Neuritic leprosy: epidemiology and therapeutic responsiveness. Lepr Rev, 1992; 63: 263-268.

14 Chehl S, Job CK, Hastings RC. Transmission of leprosy in nude mice. Am J Trop Med Hyg, 1985; 34: 1161-1166.

15 Rees RJW, McDougall AC. Airborne infection with Mycobacterium leprae in mice. J Med Microbiol, 1977; 10: 63-68. 